\title{
No Association between the Response to the Addition of an Atypical Antipsychotic Drug to an SSRI or SNRI and the BDNF (Val66Met) Polymorphism in Refractory Major Depressive Disorder in Japanese Patients
}

\author{
Reiji Yoshimura', Taro Kishi ${ }^{2,3}$, Hikaru Hori ${ }^{1}$, Atsuko Ikenouchi-Sugita', Wakako Umene-Nakano ${ }^{1}$, Asuka Katsuki ${ }^{1}$, \\ Kenji Hayashi ${ }^{1}$, Nakao Iwata ${ }^{3}$, Jun Nakamura ${ }^{1}$ \\ 'Department of Psychiatry, University of Occupational and Environmental Health, Kitakyushu, Japan, ${ }^{2}$ Department of Psychiatry Research, \\ The Zucker Hillside Hospital, New York, USA, ${ }^{3}$ Department of Psychiatry, Fujita Health University, School of Medicine, Toyoake, Japan
}

\begin{abstract}
Objective: This study examined the association between the brain-derived neurotrophic factor (BDNF) (Val66Met) polymorphism and the response to the addition of an atypical antipsychotic drug to a selective serotonin reuptake inhibitor (SSRI) or serotonin-norepinephrine reuptake inhibitor (SNRI) in treatment-refractory depression.

Methods: The study enrolled 64 patients meeting the Diagnostic and Statistical Manual of Mental Disorders-IV criteria for major depressive disorder who were treated with at least two courses of a single antidepressant, but who had Hamilton Depression Rating Scale (HAMD-17) scores $\geq 15$ points that were reduced less than $50 \%$ over at least a 4 -week treatment period. There were 24 males and 40 females (age range 27-68 years; mean $\pm S D, 48 \pm 13$ years). The patients' clinical improvement was evaluated using the HAMD-17. Patients with at least a 50\% decrease in the HAMD-17 score were defined as responders. Serum BDNF levels were assayed using enzyme-linked immunosorbent assays and the presence of the BDNF (Val66Met) polymorphism was determined using the TaqMan genotyping assay.

Results: No correlation was found between the BDNF (Val66Met) polymorphism and a positive response to adding an atypical antipsychotic drug. No differences were observed in the changes in the serum BDNF levels and HAMD-17 scores between Val66Val and Met-carriers. In addition, in patients who experienced remission, the atypical antipsychotic drug was discontinued after at least 3 months of treatment and the patients were then followed for 1 year; 14 of 27 patients (52\%) relapsed within 1 year. Conclusion: These results suggest that the BDNF (Val66Met) polymorphism is not associated with the response to the augmentation of a SSRI or SNRI with an atypical antipsychotic drug, and that the combination of an atypical antipsychotic drug and a SSRI or SNRI should be continued for 3 months or more in refractory depressed patients in the Japanese population.
\end{abstract}

KEY WORDS: Brain-derived neurotrophic factor; Treatment-resistant depressive disorder; Antipsychotic drug; Polymorphism; Selective Serotonin Reuptake Inhibitor; Serotonin Norepinephrine Reuptake Inhibitor.

\section{INTRODUCTION}

Although numerous treatments are available, only about one-third of depressed patients receiving initial antidepressant treatment achieve remission. ${ }^{1)}$ Further strategies in the treatment of resistant depression include switching to another drug of the same class or to another

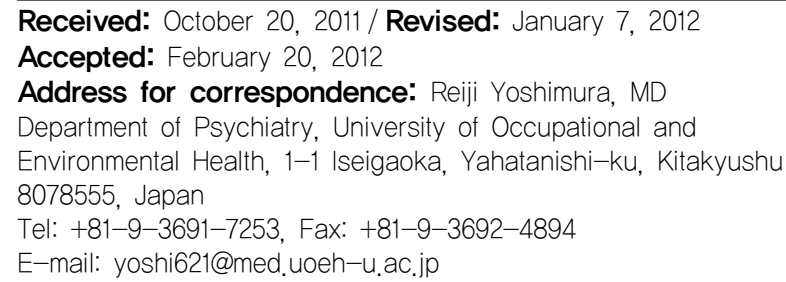

class of antidepressant. ${ }^{2}$ Lithium augmentation is the best-studied augmentation strategy. ${ }^{3)}$ Several recent meta-analyses demonstrated that atypical antipsychotic augmentation in major depressive disorder is effective and well tolerated. ${ }^{4)}$ In a preliminary study, we first demonstrated that the addition of atypical antipsychotic drugs to antidepressants or mood stabilizers increased plasma brain-derived neurotrophic factor (BDNF) levels. ${ }^{5)}$ The $B D N F$ gene is an important candidate for elucidating the mechanism of action of antidepressants or antipsychotics because BDNF plays a significant role in the functioning of the serotonin system. The human $B D N F$ gene maps to chromosome 11p13 and contains a functional 196G/A single nucleotide polymorphism (rs6265) known to cause an 
amino acid substitution from valine to methionine in exon I (Val66Met). The $B D N F$ gene encodes a precursor peptide that is cleaved proteolytically to form the mature protein BDNF. $\left.{ }^{6}\right)$ This study investigated the association between the BDNF (Val66Met) polymorphism and a positive response to the addition of an atypical antipsychotic drug to selective serotonin reuptake inhibitor (SSRI) or serotonin-norepinephrine reuptake inhibitor (SNRI) therapy in refractory depression. We also examined the natural course of the patients who achieved remission with the treatment after discontinuing the atypical antipsychoric drug.

\section{METHODS}

The study enrolled 64 patients meeting the Diagnostic and Statistical Manual of Mental Disorders-IV-TR criteria for major depressive disorder. Of these, 23 had psychotic features (psychotic depression) and the remaining $31 \mathrm{did}$ not (non-psychotic depression). There were 24 males and 40 females, ranging in age from 27 to 68 (mean \pm standard

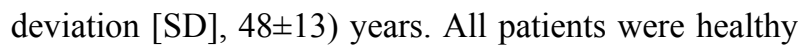
physically and free of current alcohol or drug abuse, comorbid anxiety, or personality disorders. Drug resistance was defined as a failure to respond to at least two courses of a single antidepressant medication administered at an adequate dose and duration until the patients were tolerant of the adverse effects. The patients who achieved remission were followed for 1 year after the atypical antipsychotic drug was discontinued after at least 3 months of treatment without changing the dose of the ongoing SSRI of SNRI. The study protocol was approved by the Ethics Committee of the University of Occupational and Environmental Health (Kitakyushu, Japan). All patients consented to participate after having been informed of the study's purpose. All patients were treated with an SSRI (paroxetine $[\mathrm{n}=20]$, sertraline $[\mathrm{n}=22]$, or fluvoxamine $[\mathrm{n}=8]$ ) or SNRI (milnacipran [ $\mathrm{n}=6]$ or duloxetine $[\mathrm{n}=8]$ ) for at least 4 weeks, but their scores on the Hamilton Rating Scale for Depression (HAMD-17) 7 were $\geq 15$ points and were reduced by less than $50 \%$ over the 4 -week treatment period. The daily doses $(\mathrm{mean} \pm \mathrm{SD})$ of paroxetine, sertraline, fluvoxamine, milnacipran, and duloxetine were $31.1 \pm 6.2,80.36 \pm 11.2 .4,119.1 \pm 22.9,110 \pm 17$, and $43.7 \pm 6.3 \mathrm{mg} /$ day, respectively. Atypical antipsychotic drugs were added to the ongoing SSRI or SNRI. The atypical antipsychotic drugs added were risperidone $(\mathrm{n}=17)$, aripiprazole $(\mathrm{n}=20)$, olanzapine $(\mathrm{n}=14)$, quetiapine $(\mathrm{n}=10)$, and perospirone $(\mathrm{n}=3)$, at daily doses of $1.0 \pm 0.4$, $8.8 \pm 3.1,4.6 \pm 1.3,136.3 \pm 39.6$, and $5.3 \pm 1.8 \mathrm{mg} / \mathrm{day}$, respectively. Benzodiazepines were the only hypnotics permitted, and their dosages were kept constant throughout the study period. The dosages of antidepressants and atypical antipsychotics varied among patients and were not fixed for ethical reasons. However, the doses of the antidepressants were not altered during the co-medication period. The clinical improvement of the patients was evaluated using the HAMD-17 before and every week after administering the atypical antipsychotic drug. Patients whose HAMD-17 scores decreased by $\geq 50 \%$ within 4 weeks after adding the atypical antipsychotic drug were defined as responders; those whose HAMD-17 scores decreased to 7 or less were defined as remitters; and the others were defined as non-responders.

Genomic DNA was extracted from peripheral leucocytes using a QIAamp DNA Blood Kit (QIAGEN, Tokyo, Japan) and was stored at $-20^{\circ} \mathrm{C}$ until analyzed. The presence of the BDNF (Val66Met) polymorphism was determined using the TaqMan genotyping assay (Applied Biosystems, Foster City, CA, USA). The serum samples were separated in a centrifuge and stored at $-80^{\circ} \mathrm{C}$ until assayed. The serum BDNF levels were measured using a BDNF Emax Immunoassay Kit (Promega, Madison, WI, USA), according to the manufacturer's instructions. In short, 96-well microplates were coated with anti-BDNF monoclonal antibody and incubated at $4^{\circ} \mathrm{C}$ for 18 hours. The plates were incubated in blocking buffer for 1 hour at room temperature. The samples diluted 100 -fold with assay buffer and BDNF standards were kept at room temperature with horizontal shaking for 2 hours and then washed with the appropriate washing buffer. The plates were incubated with antihuman BDNF polyclonal antibody at room temperature for 2 hours and washed with the washing buffer. Then, they were incubated with anti-IgY antibody conjugated to horseradish peroxidase for 1 hour at room temperature, and incubated again in peroxidase substrate and tetramethylbenzidine solution to induce a color reaction. The reaction was stopped with $1 \mathrm{~mol} / \mathrm{L}$ hydrochloric acid. The absorbance at $450 \mathrm{~nm}$ was measured with an Emax automated microplate reader. Measurements were performed in duplicate. The standard curve was linear from 5 to $5,000 \mathrm{pg} / \mathrm{ml}$, and the detection limit was $10 \mathrm{pg} / \mathrm{ml}$. Cross-reactivity to related neurotrophins (NT-3, NT-4, and NGF) was less than 3\%. The intra- and inter-assay coefficients of variation were $5 \%$ and $7 \%$, respectively. The recovery rate of exogenously added BDNF in the measured plasma samples exceeded $95 \%$. 


\section{Statistical analysis}

The $\chi^{2}$ test was used to compare Val/Val and Met-carriers between responders and non-responders. The level of significance for all analyses was set at $p<0.05$.

\section{RESULTS}

Of the 64 patients, 27 (42\%) responded to the addition of an antipsychotic drug to a SSRI or SNRI in refractory major depressive disorder. The Val66Met allele frequencies were in Hardy-Weinberg equilibrium $\left(\chi^{2}=0.0693\right.$, $p=0.727)$. The genotypes of the 64 patients were BDNF Val66Val, Val66Met, and Met66Met in 32.8, 53.1, and $14.09 \%$ of the subjects, respectively. There was no difference in the BDNF genotype (Val66Met) between responders and non-responders $\left(\chi^{2}=0.01840, p=0.8319\right)$ (Table 1). No differences were also found in the changes in the plasma BDNF levels $(p=0.138)$ (Fig. 1) and HAMD17 scores $(p=0.445$ ) (Fig. 2) between patients with Val66Val and Met-carriers. Of the 27 responders, $19(70 \%)$ achieved remission (HAMD-17 $\leq 7$ ) within 8 weeks after atypical antipsychotic augmentation of the SSRI or SNRI. The atypical antipsychotic drug was discontinued at least 3 months (mean \pm SD, $11.8 \pm 1.4$ weeks) after remission was achieved, and we followed the patients for up to 12

Table 1. BNDF (Val66Met) polymorphism and response

\begin{tabular}{lcc}
\hline & Val/Val & Met-carriers \\
\hline Responders & 10 & 17 (Val/Met; 13, Met/Met 4) \\
Non-responders & 11 & 26 (Val/Met; 22, Met/Met 5) \\
\hline
\end{tabular}

BDNF, brain-derived neurotrophic factor.

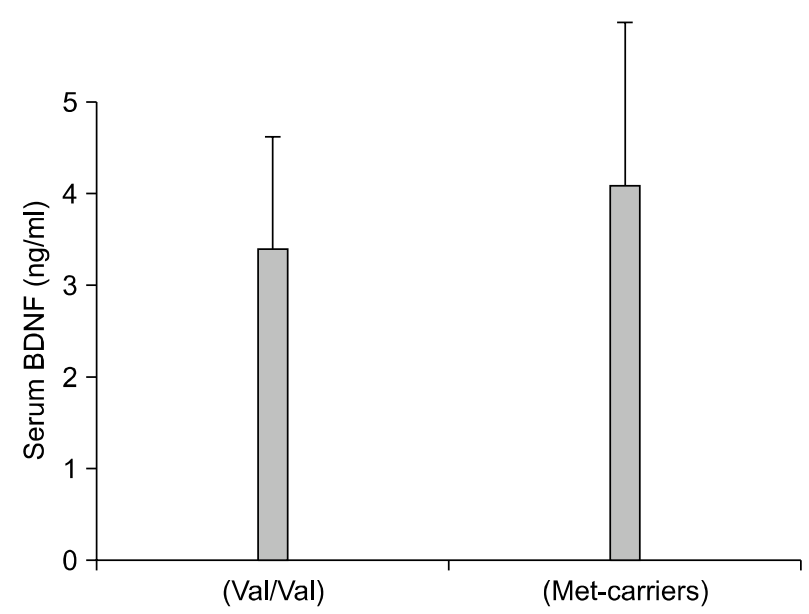

Fig. 1. Serum brain-derived neurotrophic factor (BDNF) levels in each allele. months. Thirteen of the 19 patients who achieved remission (68\%) relapsed (Fig. 3).

\section{DISCUSSION}

Previously, we reported that treatment with paroxetine or milnacipran significantly increased serum BDNF levels at 8 weeks, but not at 4 weeks. ${ }^{8}$ Furthermore, we demonstrated that risperidone did not alter the serum BDNF levels in depressed bipolar patients. ${ }^{9}$ We also found that the plasma BDNF levels in patients who responded to augmentation of SSRI or SNRI therapy with an atypical antipsychotic drug were increased 4 weeks after their treatment, while the levels in non-responders were not. The effects of atypical antipsychotic drugs on blood BDNF levels in schizophrenia remain controversial. It has been reported that olanzapine increased, ${ }^{10,11)}$ or did not change, blood BDNF levels. ${ }^{12)}$ Previously, we reported that aripi-

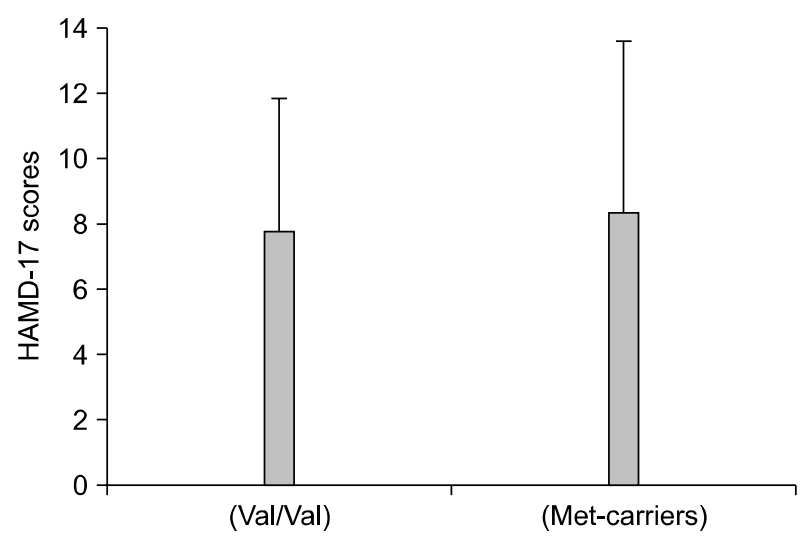

Fig. 2. Hamilton Depression Rating Scale (HAMD)-17 scores in each allele.

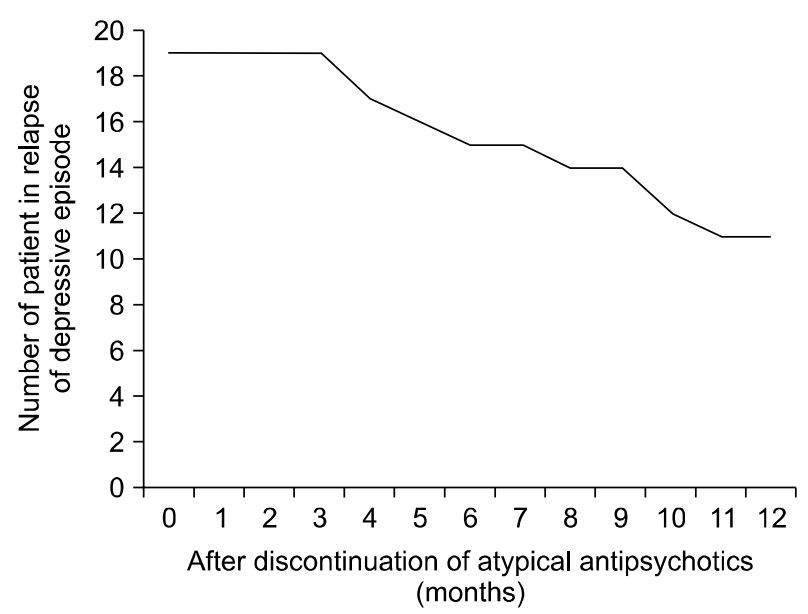

Fig. 3. The number of patients who relapsed after the discontinuation of atypical antipsychotic drugs. 
prazole slightly, but significantly, increased plasma BDNF levels in first-episode patients with untreated schizophrenia. ${ }^{13)}$ Considering these findings, we can conclude that although the effects of the atypical antipsychotic drug on the plasma BDNF levels seem to be weak, the combination of an atypical antipsychotic drug with a SSRI or SNRI might have a potent effect compared with each antidepressant alone. The mechanisms by which the combination of an antidepressant and an atypical antipsychotic drug achieves its potent effects on BDNF in depressed patients remain unknown. However, the blocking effects of 5- $\mathrm{HT}_{2 \mathrm{~A}}$ in atypical antipsychotic drugs are thought to play a role. ${ }^{14)}$

In a meta-analysis, Zou et al.$^{15)}$ demonstrated an association between the BDNF (Val66Met) polymorphism and the treatment response to antidepressants including SSRIs, SNRIs, tricyclic antidepressants, and other classes of antidepressants in patients with major depressive disorder. They found that BDNF (Val66Met) heterozygous patients had a better response rate than Val66Val homozygote patients, especially in Asian populations. However, we found no correlation between the BDNF (Val66Met) polymorphism and the response to SSRIs (paroxetine and sertraline) and the changes in serum BDNF levels. ${ }^{16)}$ Therefore, it remains controversial whether the BDNF (Val66Met) polymorphism is related to the response to antidepressants. It is possible that the class of antidepressants or the duration of treatment affected the results. To our knowledge, this is the first report to investigate the association between the BDNF (Val66Met) polymorphism and the response to atypical antipsychotic augmentation of a SSRI or SNRI, and it found no association between the two. We also examined the response and the changes in serum BDNF levels after adding an atypical antipsychotic to the SSRI or SNRI between Val66Val and Met-carriers. Another important finding of this study was that about half of the patients who recovered from a depressive state as a result of the augmentation of SSRI or SNRI therapy with an atypical antipsychotic drug relapsed within 1 year after discontinuing the atypical antipsychotic drug. This indicates that when patients have recovered from their depressive state as a result of the addition of an atypical antipsychotic drug to a SSRI or SNRI, the combined regimen should be maintained for at least 3 months.

There are several limitations to this study. First, the sample size was small and heterogeneous. Second, this was a flexibly designed, uncontrolled, open study. The possibility that the effect of the ongoing antidepressant emerged late could not be ruled out. Third, the types and doses of anti- depressants and antipsychotics differed among the patients. Therefore, further study using a larger homogeneous sample and a stricter protocol for drug administration is needed to confirm our preliminary findings.

In conclusion, no correlation was found between the response to the addition of an atypical antipsychotic drug to ongoing SSRI or SNRI therapy and the BDNF (Val66Met) polymorphism in refractory depressed patients. Moreover, when the patients enter remission after augmenting an SSRI or SNRI with an atypical antipsychotic, the atypical antipsychotic drug should be continued for at least 3 months in refractory depression in Japanese patients.

\section{REFERENCES}

1. Rush AJ, Trivedi MH, Wisniewski SR, Nierenberg AA, Stewart JW, Warden D, et al. Acute and longer-term outcomes in depressed outpatients requiring one or several treatment steps: a STAR ${ }^{*} D$ report. Am J Psychiatry 2006; 163:1905-1917.

2. Kelsey JE. Switching drug class after initial SSRI failure. J Clin Psychiatry 1997;58:326-327.

3. Crossley NA, Bauer M. Acceleration and augmentation of antidepressants with lithium for depressive disorders: two meta-analyses of randomized, placebo-controlled trials. $J$ Clin Psychiatry 2007;68:935-940.

4. Nelson JC, Papakostas GI. Atypical antipsychotic augmentation in major depressive disorder: a meta-analysis of placebo-controlled randomized trials. Am J Psychiatry 2009; 166:980-991.

5. Yoshimura R, Ikenouchi-Sugita A, Hori H, Umene-Nakano $\mathrm{W}$, Katsuki A, Hayashi $\mathrm{K}$, et al. Adding a low dose atypical antipsychotic drug to an antidepressant induced a rapid increase of plasma brain-derived neurotrophic factor levels in patients with treatment-resistant depression. Prog Neuropsychopharmacol Biol Psychiatry 2010;34:308-312.

6. Mowla SJ, Farhadi HF, Pareek S, Atwal JK, Morris SJ, Seidah NG, et al. Biosynthesis and post-translational processing of the precursor to brain-derived neurotrophic factor. J Biol Chem 2001;276:12660-12666.

7. Hamilton M. A rating scale for depression. J Neurol Neurosurg Psychiatry 1960;23:56-62.

8. Yoshimura R, Mitoma M, Sugita A, Hori H, Okamoto T, Umene $\mathrm{W}$, et al. Effects of paroxetine or milnacipran on serum brain-derived neurotrophic factor in depressed patients. Prog Neuropsychopharmacol Biol Psychiatry 2007; 31:1034-1037.

9. Yoshimura R, Nakano Y, Hori H, Ikenouchi A, Ueda N, Nakamura J. Effect of risperidone on plasma catecholamine metabolites and brain-derived neurotrophic factor in patients with bipolar disorders. Hum Psychopharmacol 2006;21:433-438.

10. Rizos EN, Papadopoulou A, Laskos E, Michalopoulou PG, Kastania A, Vasilopoulos D, et al. Reduced serum BDNF levels in patients with chronic schizophrenic disorder in relapse, who were treated with typical or atypical antipsychotics. World J Biol Psychiatry 2008:1-5.

11. González-Pinto A, Mosquera F, Palomino A, Alberich S, Gutiérrez A, Haidar $\mathrm{K}$, et al. Increase in brain-derived neurotrophic factor in first episode psychotic patients after treatment with atypical antipsychotics. Int Clin Psycho- 
pharmacol 2010;25:241-245.

12. Hori H, Yoshimura R, Yamada Y, Ikenouchi A, Mitoma M, Ida Y, et al. Effects of olanzapine on plasma levels of catecholamine metabolites, cytokines, and brain-derived neurotrophic factor in schizophrenic patients. Int Clin Psychopharmacol 2007;22:21-27.

13. Chlan-Fourney J, Ashe P, Nylen K, Juorio AV, Li XM. Differential regulation of hippocampal BDNF mRNA by typical and atypical antipsychotic administration. Brain Res 2002;954:11-20.

14. Yoshimura R, Hori H, Ikenouchi-Sugita A, Umene-Nakano $\mathrm{W}$, Katsuki A, Hayashi K, et al. Aripiprazole altered plasma levels of brain-derived neurotrophic factor and catecho- lamine metabolites in first-episode untreated Japanese schizophrenia patients. Hum Psychopharmacol 2012;27:33-38.

15. Zou YF, Ye DQ, Feng XL, Su H, Pan FM, Liao FF. Meta-analysis of BDNF Val66Met polymorphism association with treatment response in patients with major depressive disorder. Eur Neuropsychopharmacol 2010;20:535-544.

16. Yoshimura R, Kishi T, Suzuki A, Umene-Nakano W, Ikenouchi-Sugita A, Hori $\mathrm{H}$, et al. The brain-derived neurotrophic factor (BDNF) polymorphism Val66Met is associated with neither serum BDNF level nor response to selective serotonin reuptake inhibitors in depressed Japanese patients. Prog Neuropsychopharmacol Biol Psychiatry 2011;35: 1022-1025. 